14. Ferrer M, Cordoba J, Garin O, et al. Validity of the Spanish version of the chronic liver disease questionnaire (CLDQ) as a standard outcome for quality of life assessment. Liver Transplant 2006; 12: 95-104.

15. Häuser W, Schnur M, Steder-Neukamm U, Muthny FA, Grandt. Validation of the German version of the Chronic Liver Disease Questionnaire. European Journal of Gastroenterology and Hepatology 2004; 16: 599-606.

16. Assessing health status and quality-of-life instruments: attributes and review criteria. Quality of Life Research 2002; 11: 193-205.

17. Linguistic Validation Process [Internet]. Institute, MAPI
Research. 2002 [cited 2013 Jan 5]. Available from: http:// www.mapi-research-inst.com/lvprocess.asp

18. Skevington SM, Lotfy M, O'Connell KA. The World Health Organization's WHOQOL-BREF quality of life assessment: Psychometric properties and results of the international field trial. A Report from the WHOQOL Group. Quality of Life Research 2004; 13: 299-310.

19. Kumarapeli K, Seneviratne R, Wijeyaratne C. Validation of WHOQOL-BREF to measure quality of life among women with polycystic ovary syndrome (PCOS). Journal of College of Community Physicians of Sri Lanka 2006; 11: $1-10$.

\title{
The development and validation of an instrument to assess caregiver burden in cerebral palsy: Caregiver Difficulties Scale
}

\author{
C J Wijesinghe ${ }^{1}$, P Fonseka ${ }^{2}$, C G Hewage ${ }^{3}$
}

(Index words: caregiver burden, cerebral palsy, Sri Lanka, instrument, development)

\begin{abstract}
Objectives To develop a valid and reliable instrument to assess caregiver burden in caregivers of children with cerebral palsy (CP).

Methods A self-administered, multidimensional instrument - Caregiver Difficulties Scale (CDS) - was developed using a combined qualitative-quantitative approach. Items for the preliminary draft were selected from existing caregiver assessment instruments by consensus of experts or key informant interviews with caregivers and service providers of children with CP. Standard item reduction techniques based on responses of 50 caregivers were used to develop the 25 item final draft of CDS. Multidimensionality of CDS was established by exploratory factor analysis, using responses of 125 caregivers. Construct validity of CDS was confirmed by assessing correlations between CDS score and two other constructs: 'Caregiver quality of life' and 'Severity of disease in the care recipient' in a sample of 90 caregivers. Internal consistency and reliability of CDS were assessed using Cronbach's alpha and testretest reliability.
\end{abstract}

Results A new instrument (CDS) was developed with four subscales measuring caregiver's concerns for child, impact on self, support for caregiving and social and economic strain. Face validity, content validity and consensual validity of CDS was established through the process of item generation. Caregiver quality of life and severity of disease in care recipient demonstrated significant moderate to high correlations $(r \geq 0.3)$ with scores of CDS, confirming construct validity. Both internal consistency and reliability of CDS were satisfactory.

Conclusions CDS is a valid and reliable instrument to assess caregiver burden among caregivers of children with cerebral palsy in Sri Lanka.

Ceylon Medical Journal 2013; 58: 162-7

\section{Introduction}

Provision of care for children with disabilities is a daunting task due to physical and emotional demands of caregiving, changes in the social life of caregiver, economic consequences of caregiving etc. Kasuya and collegues define caregiver burden as 'a multidimensional response to physical, psychological, emotional, social and financial stressors associated with caregiving experience' [1]. Burden of caregiving can adversely affect physical and mental health of caregivers, leading to poor quality of care and unmet patient needs [1]. These effects are even more pronounced on caregivers in developing countries where basic facilities for the disabled are limited and respite

Departments of ${ }^{1}$ Community Medicine and ${ }^{2}$ Psychiatry, Faculty of Medicine, University of Ruhuna, ${ }^{3}$ Department of Community Medicine, Faculty of Medical Sciences, University of Sri Jayawardenapura, Sri Lanka.

Correspondence: CJW, e-mail:<cjw@med.ruh.ac.lk>. Received 5 June 2013 and revised version accepted 3 October 2013. Competing interests: none declared. 
care is virtually unavailable. Therefore, assessment of caregiver burden and its health impact should be an essential part of any long term care plan for children with disabilities.

Poor attention to assessment of caregiver burden in developing countries is evident by relative lack of measures designed for this purpose. Many instruments on caregiver assessment are developed in affluent countries, with different socio-cultural backgrounds. Socio-cultural factors play an important role in determining the burden of caregiving experienced by an individual $[2,3,4]$. The aim of this study was to develop a new instrument to assess caregiver burden, and capture the impact of factors which influence the process of caregiving in the local context.

Cerebral palsy (CP) is a life long, developmental disorder with a range of non-progressive motor disabilities and associated functional problems [5]. It is the commonest cause of physical disability in children in many countries and is considered as a 'prototype' of physical disabilities [6]. Therefore, an instrument developed for assessing caregivers of children with $\mathrm{CP}$ may be relevant in assessing caregivers of children with other physical disabilities.

The data on caregiver burden obtained by using this instrument can be utilized by health and social care planners to decide on the type of support services needed by the caregivers and to evaluate the effectiveness of supportive interventions. It will also help identify caregivers at risk of negative consequences, and take preventive action to improve their health and well-being.

\section{Methods}

The new caregiver assessment instrument "Caregiver Difficulties Scale" (CDS) was developed in three stages.

\section{Devising the items for the preliminary draft of CDS}

A thorough literature search was conducted to identify different dimensions of caregiver burden and existing caregiver assessment instruments. Relevant items were chosen from existing instruments through the consensus of a panel of experts (a consultant community physician, consultant psychiatrist, consultant paediatrician, consultant rheumatologist, psychologist and a sociologist), based on relevance, applicability and suitability of those items to the local setting. Selected items were then translated to Sinhala using translationback translation method.

In addition, new items reflecting caregiving experiences unique to the local context were developed through key informant interviews with principal caregivers of children with $\mathrm{CP}$ and service providers, in the Galle district. The caregivers of children with $\mathrm{CP}$ registered at "Senehasa" Children's Resource Centre (SCRC), Galle, a resource centre for children with special needs, were selected for the interviews. A purposive sample of caregivers was chosen to ensure sample heterogeneity and diversity of information on issues related to caregiving. The service providers were interviewed to explore their perspective of caregiver concerns in raising a child with a disability. Findings from these interviews were used to form a list of items describing various caregiver concerns and experiences.

Thus, a draft instrument consisting of 60 items that were considered appropriate and comprehensive, was formulated by combining the items devised by the above two methods.

\section{Formulation of subscales and response categories}

The 60 items in the initial draft of CDS were assigned to 4 subscales, according to the main area of caregiver concerns reflected by each item. Subscale 1 consisted of 15 items assessing caregivers' concerns for the child. Subscale 2 had 25 items on caregivers' concerns for self, while subscale 3 included 11 items on concerns for the family. Subscale 4 had 9 items assessing social and financial concerns of caregivers. Appropriateness of item assignment was later assessed using item analysis and factor analysis.

Each item was scored on a 5-point adjectival scale, indicating the extent or the frequency of occurrence of the condition/experience described by a particular item. A score was allocated for each response and the final score was obtained by summing up the scores of all items. Several items which required reverse scoring were included in a random fashion to identify 'a set-response' if and when it occurred.

\section{Development of the final draft of the CDS}

Initial draft of the CDS was pre-tested among a sample of 15 caregivers of children with $\mathrm{CP}$ receiving services from SCRC but who live outside Galle District. The draft instrument was then subjected to a qualitative review by a group of caregivers and service providers attending SCRC, for confirmation of its appropriateness and comprehensiveness. Assessment of frequency of endorsement and item analysis was done to reduce the number of items in the final draft, while preserving content coverage [7]. The data for item analysis was obtained from included 50 caregivers of children with $\mathrm{CP}$ registered at SCRC, but not included in previous steps of instrument development.

The final draft of the CDS included 25 items. Multidimensionality of the final draft was confirmed by exploratory factor analysis, using data obtained from a sample of 125 principal caregivers of children with CP attending SCRC, Galle. The Kaiser-Meyer-Olkin (KMO) test and Bartlett's Test of Sphericity were used to determine the appropriateness of factor analysis. A KMO statistic of 0.842 was obtained in present analysis, confirming adequacy of the sample. Bartlett's Test of Sphericity was significant (approximate chi square $=1613.835$, degree of freedom $=300, p<0.001$ ).

Principal component analysis was performed to identify the number of factors to be rotated. Six factors were extracted based on the root criterion of selecting 
factors with eigenvalues greater than 1 and the Scree test was used to examine a plot of eigenvalues. The factors with eigenvalues in the sharp descending part of the plot were retained. The six factors were rotated using Varimax rotation with Kaiser normalization. A factor loading of 0.3 was accepted as a salient loading, according to the guidelines given by Kline [8]. Table 1 shows factor loadings of individual items after Varimax rotation. (Only the factor loadings above 0.3 are shown in the table).

Thirteen pure variables (with factor loadings above 0.3 only on one factor) were identified by factor rotation. Complex variables having factor loadings of 0.3 or greater on more than one factor with a difference of $>0.2$, were assigned to the factors on which they had the highest loading. Remaining complex variables with factor loadings with a difference of $<0.2$ were assigned to the most appropriate factor on the basis of logical fit (content validity) and verified by item analysis. This procedure was helpful in preserving maximum respondent input while maintaining the content validity and interpretability of factors.

Based on item-scale correlations and factor loadings of individual items, appropriate changes were made in assignment of items to different subscales.

\section{Validation of CDS}

Psychological constructs such as caregiver burden cannot be measured using a 'gold standard' to establish criterion validity. Therefore, the validity of CDS was appraised using construct validity, by assessing the degree of correlations between the caregiver burden and the hypothetical constructs - quality of life of caregivers and severity of disease in the care recipient - using Pearson's $r$. A correlation coefficient of 0.3 was considered as the critical value for acceptance.

A sub sample of 90 principal caregivers, recruited from the sample of caregivers selected for factor analysis, was included in the validation study. Level of caregiver burden was measured using the CDS and caregiver quality of life was assessed using the validated Sinhala version of World Health Organization Quality of Life Questionnaire (WHOQOL-BREF). Severity of disease in the care recipient was assessed by a consultant paediatrician, by assigning a severity score for each care recipient, based on the child's functional level and other associated problems. Reliability of CDS was confirmed by assessing internal consistency and test-retest reliability. Internal consistency of the items in each domain was assessed using Cronbach's alpha, during item analysis. Test-retest reliability was measured by re-administering the CDS two weeks after the initial administration, to a group of 25 respondents. The two sets of data were compared using paired t test.

Approval for the study was obtained from Ethical Review Committee of Faculty of Medicine, Galle. Informed consent was obtained from the participants at all stages of the study. All the analyses during the development and validation process were conducted using SPSS version 15.0.

\section{Results}

The authors were able to develop a concise, multidimensional caregiver assessment instrument with four subscales using a combined qualitative-quantitative approach.

Fifty three items that had endorsement frequencies within the range of 0.2-0.8 were selected for item analysis. Items with higher item-total correlations were retained in the final draft of CDS. All the items retained had item-total correlations above 0.3 . A mean inter-item correlation of 0.3 or above was observed in all subscales. All except two items met item-scale correlation criterion of 0.3 or above, which was used in determining the appropriateness of assignment of items to subscales.

Principal component analysis identified six factors which accounted for $56.75 \%$ of the variance in the data set. These factors were named as "Concerns for the child", "Role limitation", "Physical impact", "Spousal support", "Family and social support" and "Economic strain". Based on factor structure and factor loadings, re-assignment of items to subscales was done while retaining the original categorization as far as possible. The modified subscales in the final version of the CDS measured caregiver's "Concerns for child", "Impact on self", "Support for caregiving" and "Social and economic strain".

As a further step in confirming that there was no major loss of information due to item reduction, the overall score of original 60 item version was compared with the score of the final draft of the CDS using Pearson's correlation coefficient. There was a near perfect correlation between the scores of two versions $(r=0.931, p<0.01)$, suggesting that the shorter version is as effective in assessing caregiver burden as the original version. The administration of CDS took between 5-10 minutes, confirming its suitability for assessing caregiver burden in a clinic or hospital setting.

Since the items in the CDS were developed from a review of existing instruments and in depth interviews with key informants, they represented common and recurring dimensions of caregiver burden. Thus, face validity, content validity and consensual validity of the instrument were confirmed by the process of item generation. All dimensions of quality of life - i.e. physical health (PHYS), psychological health (PSYCH), social relationships (SOCIAL) and environment (ENVIR) demonstrated a moderate correlation $(r \geq 0.3)$ with the total score of CDS and subscale scores, while direction of correlation was negative as hypothesized (Table 2 ). Total CDS score had a moderate correlation $(r=0.479)$ with total severity score and direction of association was positive as anticipated. Magnitude of $r$ in three subscales was above 0.3 . Severity score demonstrated good correlations with "Concerns for child" $(r=0.444)$ and "Social and economic strain" ( $r=0.450)$. However, subscale 3 (Support for caregiving) had a slightly lower correlation with severity of disease $(r=0.295)$ (Table 2$)$.

Cronbach $\alpha$ value for the total scale of CDS was 0.911 . Cronbach $\alpha$ of $0.68-0.84$ in all subscales indicated satis- 
factory internal consistency within each subscale (Table 3 ). Analysis of test-retest reliability demonstrated no significant difference between test and re-test scores of CDS.

\section{Discussion}

Generally, instruments used for caregiver assessment in many studies were psycho-social scales that measure isolated variables such as stress, depression, quality of life, social support, and family functioning [9-13]. Most of the widely used generic instruments with good psychometric properties, measure caregiver burden among specific populations such as caregivers of elderly or patients with psychiatric illness. Some items in those questionnaires were found to be inappropriate and inapplicable to the caregivers of children.

The new instrument, CDS incorporated the perceptions on caregiver burden in various other research settings, reflected in items chosen from existing instruments. In addition, it included items reflecting experiences of Sri Lankan caregivers of children with CP, derived through key informant interviews. Thus, CDS is believed to be a sufficiently comprehensive, generic instrument for assessing caregiver burden in a population of Sri Lankan caregivers of children with CP.

Multidimensional caregiver assessment instruments examine the relationship between caregiver burden and a number of areas such as caregiver health, finances, social relationships and family functioning and are believed to be superior in assessing caregiver burden, due to their high conceptual sensitivity [3]. The first two subscales of CDS, namely "Concerns for the child" and "Impact on self" included items assessing caregiver concerns about the child and family, effects of caregiving on self-care of the caregiver and execution of their roles outside caregiving. Therefore, these items collectively reflect the strain of caregiving on physical and emotional health of the caregiver and the time demands of caregiving. Subscale 3 (Support for caregiving) assesses the family and social support while subscale 4 examines the social and financial strain of caregiving. Thus, the common dimensions of caregiver burden identified in the literature are replicated in CDS, despite differences in the socio-cultural background.

Table 1. Factor loadings of individual items after Varimax rotation

\begin{tabular}{|c|c|c|c|c|c|c|}
\hline Item No. & Factor 1 & Factor 2 & Factor 3 & Factor 4 & Factor 5 & Factor 6 \\
\hline 1 & .356 & .301 & & & & \\
\hline 2 & .306 & & .420 & & & \\
\hline 3 & .592 & & & & & .311 \\
\hline 4 & .538 & & & & & \\
\hline 5 & .761 & & & & & \\
\hline 6 & .861 & & & & & \\
\hline 7 & .481 & & & & & \\
\hline 8 & .620 & & & & & \\
\hline 9 & .369 & & & .594 & & \\
\hline 10 & .312 & .590 & & .387 & & \\
\hline 11 & & & & .520 & & \\
\hline 12 & & .312 & & .675 & & \\
\hline 13 & .314 & .390 & & .330 & & \\
\hline 14 & & .464 & & .372 & & \\
\hline 15 & & & .858 & & & \\
\hline 16 & & & .841 & & & \\
\hline 17 & & & & & .612 & \\
\hline 18 & & & & & .692 & \\
\hline 19 & & .857 & & & & \\
\hline 20 & & .608 & & & & .320 \\
\hline 21 & & & & & .749 & \\
\hline 22 & .384 & .543 & & & & \\
\hline 23 & .323 & .359 & & & & .498 \\
\hline 24 & & & & & & .452 \\
\hline 25 & .525 & & & & & .576 \\
\hline $\begin{array}{l}\text { Eigenvalues } \\
\text { before rotation }\end{array}$ & 8.51 & 2.26 & 1.88 & 1.69 & 1.22 & 1.04 \\
\hline
\end{tabular}

Only the factor loadings above 0.3 are shown in the table 
Table 2. Association between measures of caregiver burden and hypothetical constructs (caregiver quality of life and severity of disease in the care recipient)

\begin{tabular}{|c|c|c|c|c|c|}
\hline Measure & CDS total & $\begin{array}{l}\text { Subscale } 1 \\
\text { (Concerns } \\
\text { for the child) }\end{array}$ & $\begin{array}{c}\text { Subscale } 2 \\
\text { (Impact on } \\
\text { self) }\end{array}$ & $\begin{array}{l}\text { Subscale } 3 \\
\text { (Support for } \\
\text { caregiving) }\end{array}$ & $\begin{array}{c}\text { Subscale } 4 \\
\text { (Social and } \\
\text { economic strain) }\end{array}$ \\
\hline 1. CDS total & - & & & & \\
\hline 2. Concerns for the child & .855 & - & & & \\
\hline 3. Impact on self & .862 & .618 & - & & \\
\hline 4. Support for caregiving & .620 & .311 & .450 & - & \\
\hline 5. Social and economic strain & .861 & .674 & .665 & .449 & - \\
\hline 6. PHYS & -.552 & -.365 & -.506 & -.433 & -.520 \\
\hline 7. PSYCH & -.663 & -.453 & -.522 & -.629 & -.618 \\
\hline 8. SOCIAL & -.596 & -.468 & -.384 & -.619 & -.529 \\
\hline 9. ENVIR & -.669 & -.502 & -.502 & -.546 & -.663 \\
\hline 10. Severity of disease & .479 & .444 & .342 & .295 & .450 \\
\hline
\end{tabular}

All correlations are significant at 0.01 level

Table 3. The internal consistency of the total scale and subscales of CDS

\begin{tabular}{lcc}
\hline Measure & No. of items & cronbach á value \\
\hline CDS total & 25 & .911 \\
Subscale 1 (Concerns for the child) & 8 & .842 \\
Subscale 2 (Impact on self) & 7 & .841 \\
Subscale 3 (Support for caregiving) & 5 & .689 \\
Subscale 4 (Social and economic strain) & 5 & .804 \\
\hline
\end{tabular}

In addition to the common stressors, the CDS includes four previously unidentified items. The two items on "opportunities for discussing problems with other family members" and "support for caregiving received from relatives and neighbours" appear to reflect the close-knit family and social relationships prevailing in our culture. Other two items on "frequent episodes of illnesses in the child" and "embarrassment experienced when traveling with the child" can be viewed as a reflection of experiences unique to a developing country where appropriate care and other resources for the disabled and societal attitudes towards disability are still in a primitive state.

Effect of caregiving on marital quality and family functioning of caregivers was a major area of concern in many studies [14-16]. However, several items covering these aspects assigned to subscale 3 had to be discarded due to high endorsement frequencies or poor correlation with the rest of the subscale. Over $80 \%$ of the respondents answered these items positively, denying any family dysfunction. This could be due to the tendency of respondents to provide "socially desirable responses" for such sensitive questions. On the other hand, strong cultural factors unique to this country may have influenced the functioning of family in a more coherent manner. There is a need for further investigation of the effects of caregiving on family functioning in the Sri Lankan context.

An increasing focus is now placed on positive aspects of caregiving, such as perception of caregiving as a worthwhile experience $[17,18]$. Although this aspect was inquired into during key informant interviews, it did not emerge as a recurring experience. The two items in the initial draft covering positive feelings of caregiving (satisfaction about caregiving and feeling of being useful and needed) had to be removed after item analysis, suggesting a poor appraisal of rewards and gratifications associated with caregiving.

Apart from these deficiencies, CDS can be regarded as a suitable instrument to assess different dimensions of caregiver burden in cerebral palsy. The fact that caregiver burden is positively related with the severity of disease in care recipient and negatively related with caregiver quality of life are well known in literature on caregiving [9, 13]. 
Results of the validation study are consistent with these findings, confirming that CDS is a valid measure of caregiver burden. However, test validation using construct validity is non-specific and is unlikely to result in very strong relationships [7]. Accordingly, correlation coefficients between the measures were in the moderate range $(r=0.3-0.6)$. Correlation between severity of disease and caregiver burden was a moderate one $(r=0.479)$, although a higher correlation was anticipated between these two constructs. It could be because, even when the number of associated problems is less, the severity of functional impairment associated with a particular problem is high resulting in heigh level of burden.

Evaluation of correlation between total CDS score and subscale scores confirmed the coherence of dimensions of the concept of caregiver burden. Correlation between total CDS score and three of the subscale scores were above 0.8 . Only subscale 3 (Support for caregiving) demonstrated a lower correlation $(0.6)$, but that too was satisfactory. Substantial correlation were observed between subscale scores, though not very high, confirming the distinct nature of the different dimensions measured by each subscale.

The relatively high internal consistency of subscales 1,2 and 4 suggest that they can be used as separate measures for assessing the different areas of caregiver concerns. This will facilitate a more detailed analysis of the burden of caregiving with a view to developing appropriate interventions. Poor performance of subscale 3 could be due to respondents' intention to give a "socially desirable response". For example, analysis of responses to individual items in that subscale revealed that nearly $80 \%$ of the participants in the validation study had responded to items on spousal support in a very positive manner while responding more negatively to other items.

The findings of this study show that the Caregiver Difficulties Scale is a valid and reliable measure for assessing caregiver burden among caregivers of children with CP receiving services from health care settings.

\section{Acknowledgements}

The authors wish to thank Prof. David L. Streiner (Research Director, Kunin-Lunenfeld Applied Research Unit and Professor, Department of Psychiatry, University of Toronto, Canada), Dr. Bilesha Perera and Dr. W. A. A. Wijayasiri (Department of Community Medicine, Faculty of Medicine, Galle) for their guidance and Dr. K. G. Somasiri (Department of Physiology, Faculty of Medicine, Galle) for granting permission to use the validated Sinhala version of WHOQOL-BREF. All consultants and academics in the expert panel that assisted in instrument development are gratefully acknowledged.

\section{References}

1. Kasuya RT, Polgar-Bailey P, Takeuchi R. Caregiver burden and burn out. Postgraduate Medicine 2000; 108: 119-23.

2. Fujiura GT. Emerging Trends in Disability. Population Reference Bureau 2008. Available from http://www.prb.org/ Articles/2001/Emerging TrendsinDisability.aspx?p=1 [Accessed 20 May, 2008]

3. Gerritson JC, van der Ende PC. The Development of a Caregiving Burden Scale. Age and Aging 1994; 23: 483-91.

4. Giunta N, Chow J, Scharlach AE, Dal Santo T. Racial and ethnic differences in family caregiving in California. Journal of Human Behaviour in the Social Environment 2004; 9: 85-109.

5. Sankar C, Mundkar N. Cerebral palsy - definition, classification, etiology and early diagnosis. Indian Journal of Paediatrics 2005; 72: 865-8.

6. Werner D. Disabled village children. A guide for community health workers, rehabilitation workers and families. Berkeley CA, Hesperian Books, 2006.

7. Streiner DC, Norman GR. Health Measurement Scales; A practical guide to their development and use. 3rd ed. New York, Oxford University Press, 2003.

8. Kline P. An easy guide to factor analysis. London, Routledge, 1994.

9. Mobarak R, Khan NZ, Munir S, et al. Predictors of stress in mothers of children with Cerebral Palsy in Bangladesh. Journal of Paediatric Psychology 2000; 25: 427-33.

10. Manuel J, Naughton MJ, Balkrishnan RF et al. Stress and adaptation in mothers with children with Cerebral palsy. Journal of Paediatric Psychology 2003; 28: 197-201.

11. Brehaut JC, Kohen DE, Raina P, et al. The health of their primary caregivers of children with Cerebral palsy: How does it compare with that of other caregivers? Paediatrics 2004; 114: e182-91.

12. Raina P, O'Donnell M, Rosenbaum P, et al. The health and well being of caregivers of children with Cerebral palsy. Paediatrics 2005; 115: e626-36.

13. Ones K, Yilmaz E, Cetinkaya B, Caglar N. Assessment of the quality of life of mothers of children with cerebral palsy (primary caregivers). Neurorehabilitation and Neural Repair 2005; 19: 232-7.

14. Cadman D, Rosenbaum P, Boyle M, Offord DR. Children with chronic illness: Family and parent demographic characteristics and psychosocial adjustment. Paediatrics 1991; 87: 884-9.

15. Senanayake MP, Mettananda DSG. Caring for a disabled child at home: Parental perceptions, Proceedings of the 9th Annual Congress of the Sri Lanka College of Paediatricians 2005; 2: 27.

16. Oelofsen N, Richardson P. Sense of coherence and parenting stress in mothers and fathers of preschool children with developmental disabilities. Journal of Intellectual and Developmental Disabilities 2006; 31: 1-12.

17. Grant G, Ramachandran P, McGrath M, et al. Rewards and gratifications among family caregivers: towards a refined model of caring and coping. Journal of Intellectual Disability Research 1998; 42: 58-71.

18. Green SE. "We are tired, not sad": benefits and burdens of mothering a child with a disability. Social Science and Medicine 2007; 64: 150-63. 